\title{
Right Ileac Fossa Mass: A Case Series
}

\author{
Kagitha Usha Rani ${ }^{1}$, Dr. R.K.Shastri ${ }^{2}$, Harika Kapu ${ }^{3}$ \\ ${ }^{1}$ Kagitha Usha Rani, Post Graduate Student Of General Surgery, Dr.Pinnamaneni Siddhartha Institute Of \\ Medical Sciences \& Research Centre, Chinnaoutapalli, Krishna Dist, Andhra Pradesh, India. \\ ${ }^{2}$ R.K. Shastri M.S., General Surgery, Professor, Department Of General Surgery, Dr.Pinnamaneni Siddhartha \\ Institute Of Medical Sciences \& Research Centre, Chinnaoutapalli, Krishna Dist, Andhra Pradesh, India. \\ ${ }^{3}$ Harika Kapu, Post Graduate Student Of General Surgery, Dr.Pinnamaneni Siddhartha Institute Of Medical \\ Sciences \& Research Centre, Chinnaoutapalli, Krishna Dist, Andhra Pradesh, India.
}

\begin{abstract}
:
Introduction: A mass in the right iliac fossa is one of the commonest problems encountered in surgical practice, requiring skill to diagnose. A clinical diagnosis is often difficult due to other conditions like obesity and guarding, with the mass being palpable only when the patient is on operating table.

Results: In the present study appendicular mass constituted 46\%, appendicular abscess 18\%, ileocaecal tuberculosis $12 \%$, carcinoma caecum $8 \%$, ovarian tumors $6 \%$, parietal lipoma $4 \%$, and retroperitoneal tumor, parietal abscess and ileocaecal tuberculosis all constituting $2 \%$ each.

Conclusion: Appendicular lump remains the most common cause for right iliac fossa mass. Ileocaecal tuberculosis is one of the most important differential diagnoses for pain abdomen in rural population.
\end{abstract}

Keywords: Right iliac fossa, appendicular abscess, tuberculosis

\section{Introduction}

Mass in the right iliac fossa is a clinical diagnostic puzzle and therapeutic dilemma for a surgeon. Despite the advances in the field of diagnosis, the surprises never cease, hence the RIF is rightly called as temple of surprises.

Mass may develop from the organs in the region or may encroach from the organs lying near to it, causing the diagnostic dilemma. The important differentials in RIF are appendicular mass/abscess, ileocecal tuberculosis and carcinoma caecum.

\section{Case Presentation:}

Case report 1:

A $10 \mathrm{yr}$ old kid presented with a 3day history of shifting pain from umbilicus to RIF with 2 episodes of vomiting's. No history of fever or any bowel disturbances. O/E: Fullness in the right iliac fossa on inspection, Tender mass measuring $8 \times 9 \mathrm{~cm}$ and ill-defined margins. Hb: 11, WBC: 9000. Erect Abdomen X-Ray: NAD. USS abdomen: Focal peritonitis with ruptured appendix. Patient was posted for appendectomy.

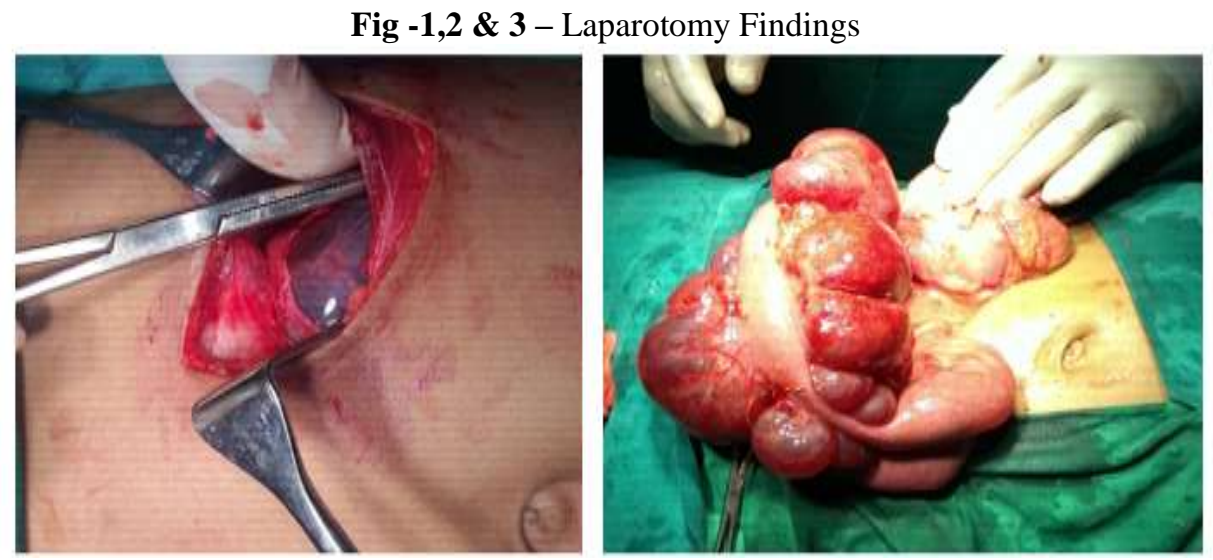




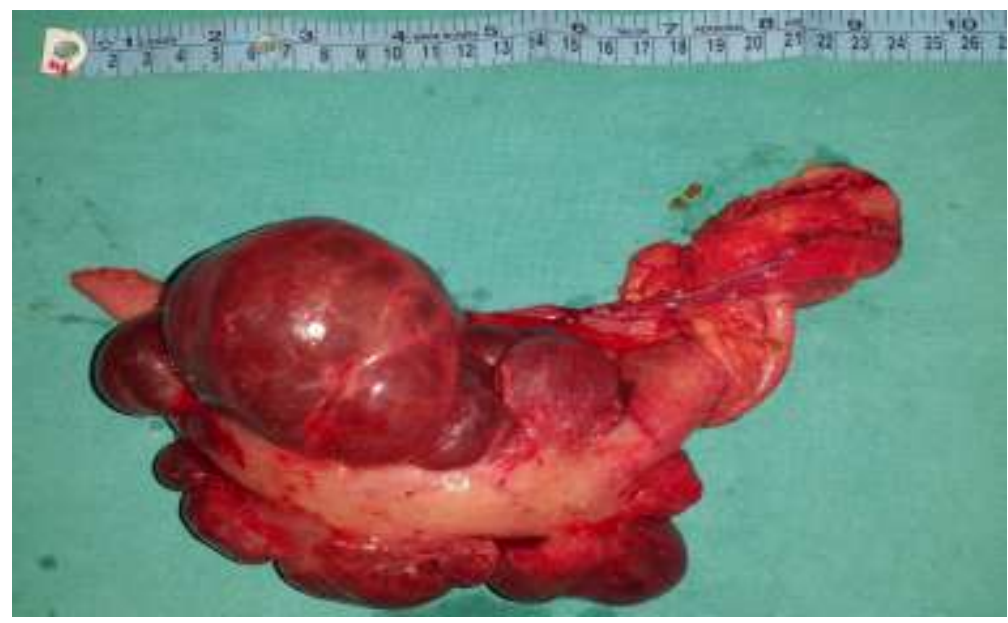

\section{Histopathology:}

Cyst filled with hemorrhagic fluid, loss of mucosal folds of distal ileum, walls lined by flattened epithelium with smooth muscle coat, lymphocytes and congested blood vessels Features are suggestive of nonspecific ileitis, non-specific lymphadenitis \& multilocular cystic lymphangioma

\section{Case report 2:}

A 25yr old male, tailor by occupation presented with a week H/O RIF pain and fever, with no h/o vomiting's or altered bowel or bladder habits. No loss of weight or appetite. O/E abdomen was tender in RIF, with no guarding or rebound tenderness. USG abd: Impression was possible acute appendicitis. Patient was advised admission but he was reluctant and self-discharged himself. Presented back in 2 days with mass in RIF and ongoing pain and fever. O/E: Ill-defined mass in RIF, tenderness present, with normal bowel sounds.

\section{Investigations:}

- Blood tests: WBC- $11000, \mathrm{Hb}-12$

ESR - 60

- USG abd: Features suggestive of appendicular abscess

- Laparotomy findings: Intestines studded with tubercles No Abscess, appendix healthy Biopsy sent for histopathology

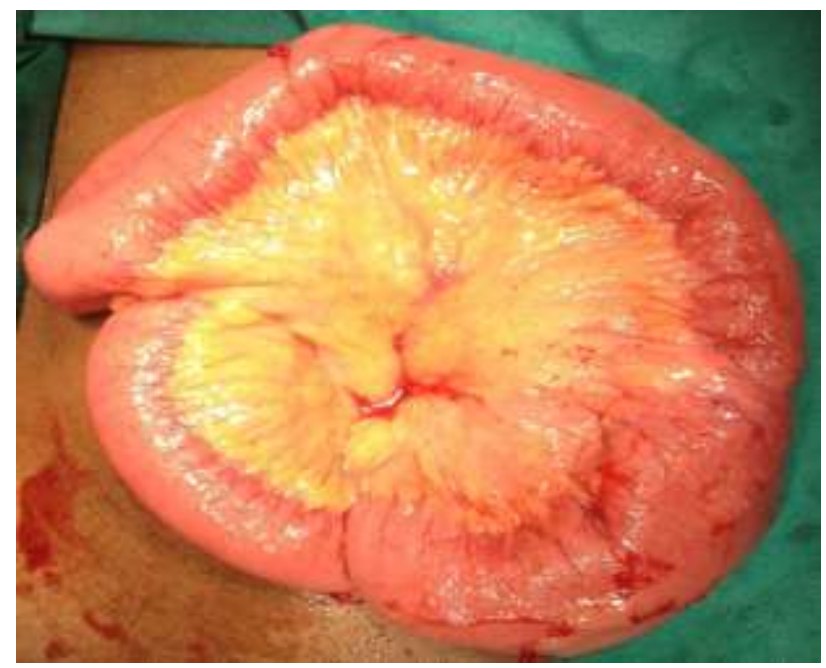

Fig-4: Intestine studded with tubercles.

Histopathology:

Epitheloid cell granuloma with central caseous necrosis noticed in the lymph node, Koch's etiology. 


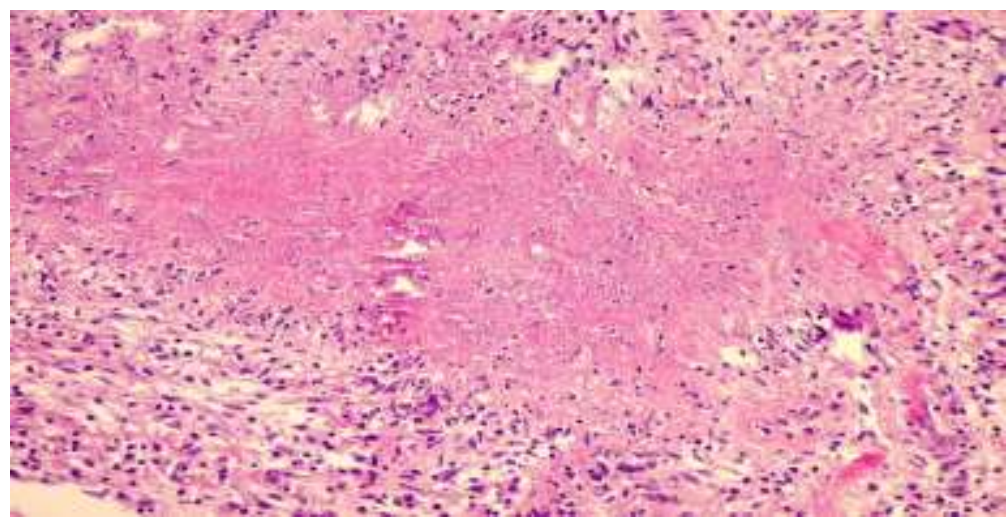

Fig-5: Koch's Etiology

\section{Case report 3:}

A 60yr old man, farmer by occupation, presented with 1 month h/o vague right sided abdominal pain, with loss of appetite, no loss of weight, no altered bowel or bladder habits. Abdominal fullness was noticed since 15 days, with no obstructive symptoms.

$\mathrm{O} / \mathrm{E}$ abdomen is soft, vertically oval lump measuring $10 \times 8 \mathrm{~cm}$ noted in the RIF, minimally tender, well defined borders.

\section{Investigations:}

- $\mathrm{Hb}-9.7 \mathrm{mg} \%$, Wbc- 9000

- Abdomen X-Ray- NAD.

- USG abd: Mass arising from caecum, ?ca caecum

\section{Laparotomy:}

- CECT abdomen \&pelvis: carcinoma caecum

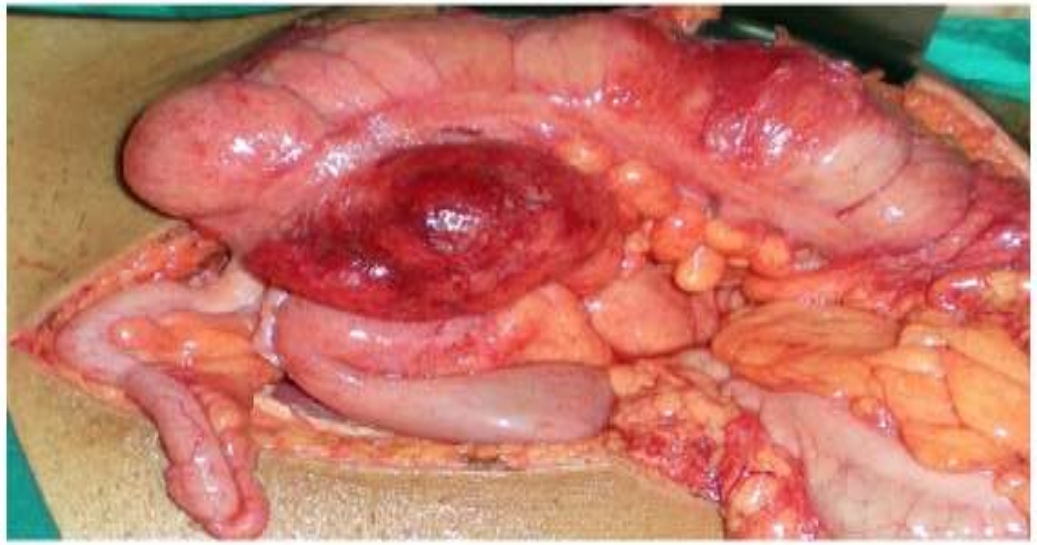

Fig-6: Carcinoma of Caecum

\section{Histopathology:}

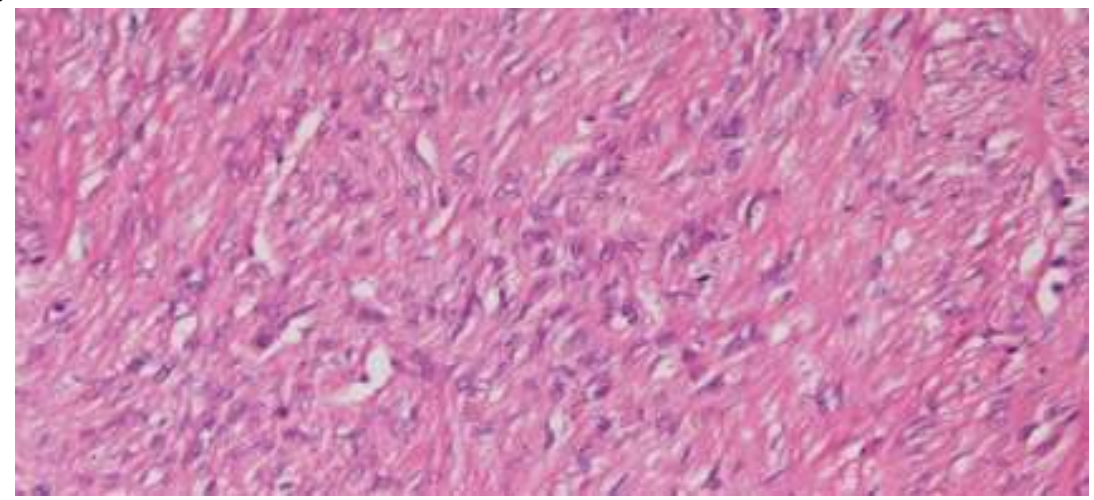

Fig-7: Spindle shaped cells 


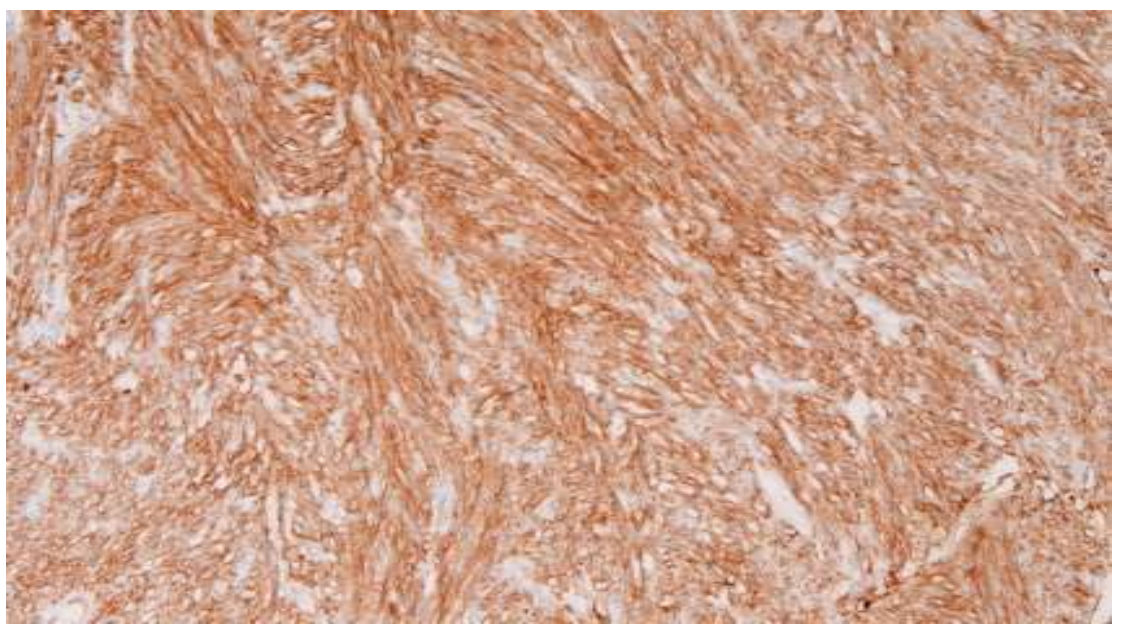

Fig-8: C-kit staining positive

\section{Discussion}

Although appendicular lump forms the majority of abdominal masses, ileocecal tuberculosis forms the 2nd most common differential in the developing countries, followed by caecal malignancies. In a prospective study of Right iliac fossa masses carried out during the period of 08/2014-08/2016, in PSIMS \&RF, about $55 \%$ of cases were appendicular masses, $23 \%$ accounted for intestinal tuberculosis, $16 \%$ of carcinoma caecum and ascending colon and $6 \%$ accounted for other conditions. Females had an increased incidence of caecal carcinoma.

Almost all cases of appendicular mass presented within 15days, in 93\% the commonest presenting symptom was abdominal pain with fever. In carcinoma of the cecum and ileocecal tuberculosis, most of them presented within 3-6 months, $75 \%$ of them presented with mass per abdomen and weight loss with vomiting's.

All cases of appendicular mass underwent conservative management and interval appendicectomy, and appendicular abscess underwent drainage procedures. $84 \%$ of the ileocecal tuberculosis underwent definitive surgery like right hemicolectomy, and rest managed with ATT after confirming the diagnosis by tissue biopsy

Abdominal ultrasonography is the imaging modality of first choice in patients presenting with a right iliac fossa mass although the specificity is low.

Surgery is the mainstay of treatment and, when done with adequate preparations, has good prognosis. Ileocecal tuberculosis is one of the most important differential diagnoses for chronic abdominal pain in the rural population. Mesenteric cysts are rare occurrences, the commonest site being distal ileum, mostly presents as mass or pain in the abdomen.

\section{Conclusion}

The Patients are under regular followup for past 4yrs and is disease free. Latest CT abdomen showed normal study except few sub centimetric nodes in mesentery and in retroperitoneum. Though the presentation was clinically insignificant further investigations turned out to show one of the rare causes of lymphoproliferative disorder. Diagnosis of this disease is based on histopathological examination. The prognosis of unicentric castle man disease is very good, close to $100 \%$ disease free survival rate by 5 years.

\section{Bibliography:}

[1]. Das S. Examination of abdominal lump, Chapter 35 In: A manual on clinical surgery. 11th edn. 2015.

[2]. Shashikala V, Alister J Victor, Sonia Rani P. B. Right iliac fossa mass: A prospective study. International Journal of Biomedical and advance Research 2016; 7(8): 388-392.

[3]. S K Shetty, M Shankar. A Clinical Study Of Right Iliac Fossa Mass. The Internet Journal of Surgery. 2013 Volume 30 Number4.

[4]. Howell S and Knapton PJ. Ileo-caecal tuberculosis. Gut 1964; 5:524-529.

[5]. Amin MA, Khan MA, Ayub M, Mohamood M, Ashraf M, Choudhry AR. Delay in the diagnosis and prognosis of caecal carcinoma - A study of 20 cases. J Ayub Med Coll Abbottabad 2001 Apr-Jun; 13(2):28-31.

[6]. Juniorsundresh.N, Narendran.S,Ramanathan.M. Evaluation of Pathological nature of the right iliac fossa mass and its management. J Biomed Sci Res. 2009; Vol 1 (1): 55-58

[7]. Arshad Kamal, Shafiullah. Significance Of Total Leucocyte Count In The Diagnosis Of Acute Appendicitis In Children. Gomal Journal of Medica Sciences January-June 2011; Vol. 9(1):90-9. 